\title{
BMJ Open Improving the identification of people with dementia in primary care: evaluation of the impact of primary care dementia coding guidance on identified prevalence
}

\author{
Paul Russell, ${ }^{1}$ Sube Banerjee, ${ }^{2}$ Jen Watt, ${ }^{3}$ Rosalyn Adleman, ${ }^{4}$ Belinda Agoe, ${ }^{5}$ \\ Nerida Burnie, ${ }^{6}$ Alex Carefull, ${ }^{7}$ Kiran Chandan, ${ }^{8}$ Dominie Constable, ${ }^{9}$ \\ Mark Daniels, ${ }^{10}$ David Davies, ${ }^{11}$ Sid Deshmukh, ${ }^{12}$ Martin Huddart, ${ }^{1}$ Ashrafi Jabin, ${ }^{13}$ \\ Penelope Jarrett, ${ }^{14}$ Jenifer King, ${ }^{15}$ Tamar Koch, ${ }^{11}$ Sanjoy Kumar, ${ }^{1}$ \\ Stavroula Lees, ${ }^{16}$ Sinan Mir, ${ }^{17}$ Dominic Naidoo, ${ }^{6}$ Sylvia Nyame, ${ }^{18}$ \\ Ryuichiro Sasae, ${ }^{18}$ Tushar Sharma, ${ }^{19}$ Clare Thormod, ${ }^{20}$ Krish Vedavanam, ${ }^{21}$ \\ Anja Wilton, ${ }^{22}$ Breda Flaherty ${ }^{23}$
}

To cite: Russell $P$,

Banerjee S, Watt J, et al. Improving the identification of people with dementia in primary care: evaluation of the impact of primary care dementia coding guidance on identified prevalence. BMJ Open 2013;3:e004023. doi:10.1136/bmjopen-2013004023

- Prepublication history and additional material for this paper is available online. To view these files please visit the journal online (http://dx.doi.org/10.1136/ bmjopen-2013-004023).

Received 14 September 2013 Revised 21 October 2013 Accepted 20 November 2013

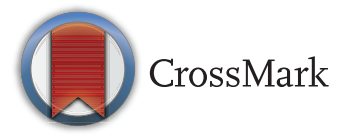

For numbered affiliations see end of article.

Correspondence to Professor Sube Banerjee; s.banerjee@bsms.ac.uk

\section{ABSTRACT}

Objective: Improving dementia care is a policy priority nationally and internationally; there is a 'diagnosis gap' with less than half of the cases of dementia ever diagnosed. The English Health Department's Quality and Outcomes Framework (QOF) encourages primary care recognition and recording of dementia. The codes for dementia are complex with the possibility of underidentification through miscoding. We developed guidance on coding of dementia; we report the impact of applying this to 'clean up' dementia coding and records at a practice level.

Design: The guidance had five elements: (1) identify Read Codes for dementia; (2) access QOF dementia register; (3) generate lists of patients who may have dementia; (4) compare search with QOF data and (5) review cases. In each practice, one general practitioner conducted the exercise. The number of dementia QOF registers before and after the exercise was recorded with the hours taken to complete the exercise.

Setting: London primary care.

Participants: 23 (85\%) of 27 practices participated, covering 79312 (19 562 over $65 \mathrm{~s}$ ) participants.

Outcomes: The number on dementia QOF registers; time taken.

Results: The number of people with dementia on QOF registers increased from 1007 to $1139\left(\chi^{2}=8.17\right.$, $p=0.004$ ), raising identification rates by $8.8 \%$. It took $4.7 \mathrm{~h}$ per practice, on an average.

Conclusions: These data demonstrate the potential of a simple primary care coding exercise, requiring no specific training, to increase the dementia identification rate. An improvement of $8.8 \%$ between 2011 and 2012 is equivalent to that of the fourth most improved primary care trust in the UK. In absolute terms, if this effects were mirrored across the UK primary care, the number of cases with dementia identified would rise by over 70000 from 364329 to 434488 raising the

\section{Strengths and limitations of this study}

- The general practitioners (GPS) who took part in this exercise were highly motivated and had been selected to participate in the National Health Service London dementia fellowship programme.

- Generalisability may be limited from these London practices to those in the rest of the UK.

- However, the coding systems used and GP information systems vary little so the demonstration that this exercise worked in practices across London may point its being applicable across the UK.

- We achieved a high participation rate and the practices covered a large population (179 312) over a wide and sociodemographically varied set of areas spread across 19 boroughs.

- The protocol generated was simple and required no training.

recognition rate from $46 \%$ to $54.8 \%$. Implementing this exercise appears to be a simple and effective way to improve recognition rates in primary care.

\section{BACKGROUND}

Dementia is one of the most common and serious disorders in later life with a prevalence of $5 \%$ and an incidence of $2 \%$ per year in people over 65 years of age. ${ }^{12}$ In the UK, estimates suggest that there are 800000 people with dementia currently. ${ }^{3}$ It causes irreversible decline in global intellectual, social and physical functioning. Abnormalities in behaviour, insight and judgement are part of the disorder, as are neuropsychiatric symptoms such as psychosis, anxiety and depression. The economic 
cost of caring for people with dementia is immense. In the $\mathrm{UK}$, the costs of dementia are around $£ 17$ billion/year, ${ }^{3}$ with some suggesting this as more than stroke, heart disease and cancer. ${ }^{4}$ More importantly, the negative impacts of dementia on those with the disorder, in terms of deteriorating function, and on carers ${ }^{56}$ are profound. There are 35 million people with dementia worldwide and this costs $\$ 600$ billion/year, with these numbers set to double and the costs to at least triple in the next 20 years. $^{78}$

The need to improve care for people with dementia is a policy priority nationally and internationally. ${ }^{9-12}$ One common finding in analyses of health systems with respect to dementia is that there is a 'diagnosis gap' in dementia with less than a half of those with dementia ever attracting a diagnosis of dementia. Also, such diagnosis and contact often occur late in the illness and/or in crisis when opportunities for harm prevention and maximisation of quality of life have passed. A common element of health strategies to improve the quality of care of people with dementia is the injunction that diagnosis rates should be increased and that diagnosis should be 'early' or 'timely'.

Part of the Department for Health for England's response to this has been to encourage primary care recognition and recording of dementia through its Quality and Outcomes Framework (QOF).$^{13}$ This is designed to incentivise, via payment, primary care services to improve the quality of care by standardising improvements in the delivery of primary medical services. The relevant indicators for 2013/2014 include:

DEM001 The contractor establishes and maintains a register of patients diagnosed with dementia.

The number of people with dementia identified in these registers compared with projected numbers from the local age and gender structure has been used as a marker of progress against the goals of the National Dementia Strategy using a commissioned dementia prevalence calculator. ${ }^{14}$ Using this methodology, figures from the Alzheimer's Society suggest that diagnosis rates in Britain range from $32 \%$ in the East Riding of Yorkshire to $76 \%$ in Belfast. ${ }^{15}$

These dementia registers rely on how dementia is coded in primary care record systems. This is generally via Read Codes which are a coded thesaurus of clinical terms that has been used within primary care in the National Health Service (NHS) since 1985. The codes available for dementia are complex with the possibility of underidentification through miscoding. NHS London therefore developed guidance on the coding of dementia (Web appendix 1). In this paper, we report the impact of applying this guidance in a sample of general practitioner (GP) practices across London to investigate whether it is possible to raise diagnosis rates through undertaking an exercise to "clean up' dementia coding and records at a practice level.

\section{METHOD}

A coding exercise protocol was developed to enable GPs to conduct a review of practice records and to recode cases where needed. This was developed by PR and SB iteratively with key stakeholders, facilitated by NHS London co-ordinated by JW. The protocol is presented in full in Web appendix table 1. This consisted of a simple five-point process.

Step 1: Identify commonly used Read Codes for dementia/memory concerns

Step 2: Obtain practice's QOF dementia register

Step 3: Run searches to generate lists of patients who may have dementia

Step 4: Compare search results with QOF dementia register

Step 5: Discuss with patients for further review

Participating practices were drawn from boroughs across London which had a GP on the 2012/13 NHS London GP Dementia Fellowship Programme. The exercise was completed by the GP on the programme in each practice as set out in Web appendix table 1. The number of patients identified with dementia in the practice QOF registers before and after the exercise was recorded as were the hours taken to complete the exercise.

\section{RESULTS}

\section{Sample}

Twenty-three $(85 \%)$ of the 27 practices available participated. These came from 19 boroughs across London. The participating practices had a practice population of 179312 with $19562(10.9 \%)$ patients over the age of 65 . The mean practice size was 8296 (range 2543-16 700). In terms of representativeness of the sample, this proportion over 65 years was similar to the overall percentage for London (11.2\%); the London rate is lower than that for the UK as a whole $(16.9 \%)$.

\section{Impact of the intervention}

The number of people in the practices' dementia QOF registers before the intervention was 1007; after the intervention there were 1139 cases on the registers (practice population 179312 with 19562 aged 65+). This was a statistically significant increase $\left(\chi^{2}=8.17\right.$, $\mathrm{p}=0.004)$. The mean number of people in the practices' dementia QOF registers before the intervention was 44 (range 0-232, SD 49). After the intervention there was an average of 50 cases on the registers (range $0-248$, SD 54 ); this was a statistically significant difference between those means $(\mathrm{t}=3.52 ; \mathrm{p}<0.001)$. Taking the numbers aged $65+$ in each practice as the denominator, this represents an identified prevalence of $5.1 \%$ before the intervention and $5.8 \%$ after. Using the most recent $\mathrm{DH}$ estimates,${ }^{14}$ the expected number of people with dementia in London is 69849 which equates to a prevalence of $8.11 \%$ of those aged $65+$. We used this to calculate the identification rate before and after the intervention. After the intervention, $71.8 \%$ of cases of dementia were 
identified compared with $63 \%$ before intervention. The effect of the intervention was therefore to increase the rate of identification of dementia in this sample by $8.8 \%$.

\section{Cost of the intervention}

The only cost of the intervention was the person time spent on the exercise which took an average of $4.7 \mathrm{~h}$ per practice.

\section{DISCUSSION}

The data presented here demonstrate the potential of a simple primary care coding exercise, requiring no specific training, to increase the rate of identification of cases of dementia. In this sample of GP practices in London, an $8.8 \%$ improvement was achieved at a cost of an average $4.7 \mathrm{~h}$ of GP time.

\section{Size of effect}

We need to consider the meaning of the $8.8 \%$ increase observed. In their recent analysis of identification rates from QOF registers across the UK, ${ }^{15}$ the Alzheimer's Society calculated for each Primary Care Trust (PCT) area (the forerunners to current Clinical Commissioning Groups (CCGs)) identification rates in the same way that we have here. They did this for 2011 and 2012 and calculated changes in numbers and rank between 2012 and 2011. In rank terms, for a PCT, an improvement of $8.8 \%$ between 2011 and 2012 would have resulted in that PCT being the fourth most improved PCT in the UK (displacing Torbay that achieved an $8.2 \%$ improvement). In terms of ranking, an improvement of $8.8 \%$ would have moved a PCT up to an indicative 68 places (of 178). In absolute terms, if this were implemented across the UK primary care with the same aggregate effect, the number of cases with dementia identified in QOF registers would rise by over 70000 from 364329 to 434488 , raising the recognition rate from $46 \%$ to $54.8 \%$.

\section{Reasons for coding error}

Implementing this exercise appears to be a simple and effective way to improve recognition rates in primary care. Completing the exercise also sheds some light on the reasons for the current coding errors. Part of this may be a wish to avoid labelling patients as having dementia on the QOF register unless there is a high level of certainty as to the diagnosis. This is an understandable and reasonable concern, as there is a stigma associated with the diagnosis, and professional attitudes to and confidence in the management of dementia may play a role in perceived underrecognition. However, all the cases reassigned in this exercise were those with an established dementia. The issues seemed more a function of computer and coding systems. This points to the potential value of this exercise. Ninety-eight per cent of London practices use Egton Medical Information Systems (EMIS) or Vision systems; these require coding by V2-5byte Read Codes. In this system, there are a large number of potential dementia-related Read Codes that might be used and these are not presented in a way that is user friendly (Web appendix 1). In this exercise, we sought to address this by operationalising how these codes should be used. Equally, the letters received by GPs from secondary care services where diagnoses may have been made were often not clear in terms of diagnosis and diagnostic category. Letters from psychiatric services were often long with the diagnosis hidden in the text. We also found cases where the first assessment letter referred to 'probable dementia' but where the definitive diagnosis in a letter 3-6 months later had not led to the coding being brought up to date. The use of multiple systems and unfamiliar terminologies by secondary care leads to confusion and lack of clarity in coding within primary care. The simple expedient of all secondary care clearly stating the International Classification of Diseases (ICD) 10 codes or the most appropriate Read code to use would address this coding problem.

\section{Limitations}

This is a simple pragmatic study and the limitations of this exercise and the data presented here need to be considered. This was not a random sample of practices or of GPs. The GPs who took part in this exercise were highly motivated and had been selected to participate in the NHS London dementia fellowship programme. They were therefore interested in dementia and also built skills during the programme within which the exercise was completed. The main likely impact of this may have been to enable them to complete the exercise more quickly than the average GP. However, even if the exercise took twice the time, this would mean that it could be achieved in a single day. The generalisability of the practices within which the exercise was completed also needs to be considered. All were group practices and they were similar to the rest of London in terms of age structure; however, the practices studied had a higher than average recognition rate before the exercise $(63 \%)$ compared with the UK as a whole $(46 \%)$. This may reflect the interests of the participating GPs and their effect on coding in those practices. This is of interest because this suggests that we were intervening in practice systems that may have been more optimised than others might be. It is quite possible that in less optimised systems there might be greater levels of coding error and so an even greater effect might be achieved. As an estimate of effect, our results might therefore be considered conservative. There will be differences between London practices and those in the rest of the UK as evidenced by the difference in age structure in London compared with the UK as a whole. However, the coding systems used and GP information systems vary less, and so the demonstration that this exercise worked in practices across London may point its being applicable across the UK. Different countries have different systems and it is likely that the detail of the approach 
here will only apply to the UK; however, the general issue of clarity in coding is likely to be of importance internationally. There are strengths in the approach used. We achieved a high participation rate and the practices covered a large population (179 312) over a wide and sociodemographically varied set of areas spread across 19 boroughs. Also, the protocol generated was simple and required no training. The data here represent a test of the feasibility of conducting the exercise and of the content of the protocol.

\section{CONCLUSIONS}

It is important to be clear that this coding exercise would form only a part of the comprehensive whole system effort to improve dementia care and to lift diagnosis rates through initiatives such as education and training, memory service commissioning, and service improvement. ${ }^{16}$ However, the results presented here suggest that completing this exercise could provide a simple, cheap and useful first step to improve accuracy of records. More accurate information can help to improve the management of patients and also help to close the diagnosis gap. ${ }^{11}$ In terms of next steps, evaluations of the effect of coding exercise in a more representative sample of practices nationwide would be of use. But equally, given its simplicity and low cost, it may be that practices and CCGs would wish to implement this without needing further evaluation other than auditing local effects. In this paper, we have provided all the materials needed to carry out the exercise.

\section{Author affiliations}

${ }^{1}$ General Practice, Waltham Forest CCG, London, UK

${ }^{2}$ Brighton and Sussex Medical School, University of Sussex, Brighton, UK

${ }^{3}$ UCL Partners, London, UK

${ }^{4}$ General Practice, Barnet CCG, London, UK

${ }^{5}$ General Practice, Haringey CCG, London, UK

${ }^{6}$ General Practice, Kingston CCG, London, UK

${ }^{7}$ General Practice, Camden CCG, London, UK

${ }^{8}$ General Practice, Bromley CCG, London, UK

${ }^{9}$ General Practice, Sutton CCG, London, UK

${ }^{10}$ General Practice, Ealing CCG, London, UK

${ }^{11}$ General Practice, Islington CCG, London, UK

${ }^{12}$ General Practice, Bexley CCG, London, UK

${ }^{13}$ General Practice, Tower Hamlets CCG, London, UK

${ }^{14}$ General Practice, Lambeth CCG, London, UK

${ }^{15}$ General Practice, Hackney CCG, London UK

${ }^{16}$ General Practice, Richmond CCG, London, UK

${ }^{17}$ General Practice, Hammersmith and Fulham CCG, London, UK

${ }^{18}$ General Practice, Greenwich CCG, London, UK

${ }^{19}$ General Practice, Southwark CCG, London, UK

${ }^{20}$ General Practice, Newham CCG, London, UK

${ }^{21}$ General Practice, Brent CCG, London, UK

${ }^{22}$ General Practice, Lewisham CCG, London, UK

${ }^{23}$ Division of Medical Education, Brighton and Sussex Medical School, Brighton, UK

Acknowledgements The authors would like to thank the staff of all the practices who participated for their help in carrying out this project.
The National Health Service London coding guidance and exercise are available on the South West Dementia Partnerships website at: http://www. dementiapartnerships.org.uk/diagnosis/resource-pack/9-coding/

Contributors All authors were involved in the design and conduct of the project; all but JW and SB carried out the exercise and collected the data. All participated in the editing of the paper and all agreed to the final content. PR and SB generated the coding protocol. Analyses were carried out by SB who also wrote the first draft of the paper. SB is the guarantor.

Funding The project was supported by the National Health Service London as part of the NHS London Dementia Fellowship Programme.

Competing interests SB has received consultancy fees, speakers' fees, research funding or educational support to attend conferences from pharmaceutical companies involved in the manufacture of antidepressants and antidementia drugs, and has been employed by the Department of Health for England. JW has been employed by National Health Service London.

Provenance and peer review Not commissioned; externally peer reviewed.

Data sharing statement All data available are used in this paper.

Open Access This is an Open Access article distributed in accordance with the Creative Commons Attribution Non Commercial (CC BY-NC 3.0) license, which permits others to distribute, remix, adapt, build upon this work noncommercially, and license their derivative works on different terms, provided the original work is properly cited and the use is non-commercial. See: http:// creativecommons.org/licenses/by-nc/3.0/

\section{REFERENCES}

1. Hofman A, Rocca WA, Brayne C, et al. The prevalence of dementia in Europe. Int J Epidemiol 1991;20:736-48.

2. Launer LJ, Brayne C, Dartigues J-F, et al. Epilogue. Neuroepidemiology 1992;11(Suppl 1):119-21.

3. Knapp M, Prince M, Albanese E, et al. Dementia UK: the full report. London: Alzheimer's Society, 2007.

4. Lowin A, Knapp M, McCrone P. Alzheimer's disease in the UK: comparative evidence on cost of illness and volume of research funding. Int J Geriatr Psychiatry 2001;16:1143-8.

5. Murray J, Schneider J, Banerjee S, et al. EUROCARE: a cross-national study of co-resident spouse carers for people with Alzheimer's disease: II-a qualitative analysis of the experience of caregiving. Int J Geriatr Psychiatry 1999;14:662-7.

6. Schneider J, Murray J, Banerjee S, et al. EUROCARE: a cross national study of co-resident spouse carers for people with Alzheimer's disease: I-factors associated with carer burden. Int J Geriatr Psych 1999;14:651-61.

7. Prince M, Jackson J. World Alzheimer's Report 2009. London, UK: Alzheimer's Disease International, 2009.

8. Wimo A, Prince M. World Alzheimer's Report 2010: the global economic impact of dementia. London, UK: Alzheimer's Disease International, 2010.

9. Department of Health. National service framework for older people. London: $\mathrm{DH}, 2001$.

10. DH. Everybody's business. London: CSIP, 2005.

11. Department of Health. Living well with dementia, a national dementia strategy. London, UK: Stationery Office, 2008.

12. NAO. National Audit Office Report. Improving services and support for people with dementia $\mathrm{HC} 604$, report by the Comptroller and Auditor General, session 2006-2007. TSO: London, 2007.

13. http://www.nhsemployers.org/Aboutus/Publications/Documents/ qof-2013-14.pdf

14. http://www.dementiapartnerships.org.uk/diagnosis/dementiaprevalence-calculator/

15. Alzheimer's Society. Mapping the dementia gap 2012; progress on improving diagnosis of dementia 2011-2012. London: Alzheimer's Society, 2012. http://www.alzheimers.org.uk/site/scripts/download info.php?filelD $=1348$

16. Banerjee S. Living Well with Dementia - development of the National Dementia Strategy for England. Int J Geriatr Psych 2010;25:917-25. 